\title{
Nuclear Reaction Dynamics Research at the Australian National University
}

\author{
D.J. Hinde ${ }^{1, a}$, M. Dasgupta ${ }^{1}$, I.P. Carter ${ }^{1}$, K.J. Cook $^{1}$, M. Evers ${ }^{1}$, D.H. Luong ${ }^{1}$, K. Ramachandran ${ }^{1, b}$, D. Rafferty ${ }^{1}$, C. \\ Simenel ${ }^{1}$, A. Wakhle ${ }^{1}$, and E. Williams ${ }^{1}$ \\ ${ }^{1}$ Department of Nuclear Physics, RSPE, Australian National University, ACT 0200, Australia
}

\begin{abstract}
This contribution presents an overview of the research program in nuclear reaction dynamics at the Australian National University.
\end{abstract}

\section{Introduction}

The research program in nuclear reaction dynamics at the Australian National University has centred around the goal of obtaining a deep understanding of the many interconnected facets of nuclear fusion. In the last 20 years, the understanding of fusion of heavy nuclei has undergone a revolution, with the influence of the identity and nuclear structure properties of the colliding nuclei now understood to play a very important role- not only at the time of contact, but also in the subsequent evolution towards a compact compound nucleus. This has resulted from both experimental and theoretical research. Aspects of current theoretical reaction dynamics research at the ANU are described in the contribution to these proceedings by $\mathrm{C}$. Simenel et al.

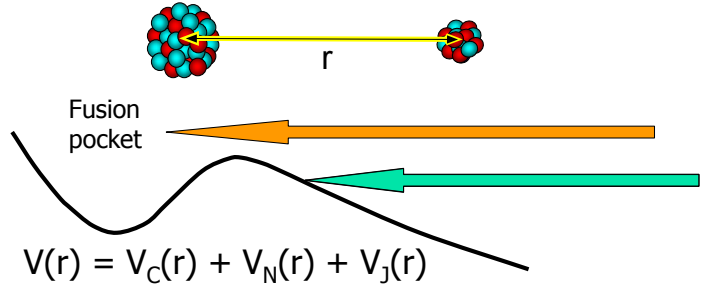

Figure 1. The barrier passing picture of fusion, showing energy as a function of radial separation of the two colliding nuclei.

The fundamental principle in describing fusion is the barrier-passing concept. The first step is to calculate the barrier itself. In principle this is achieved by adding the multitude of individual nucleon-nucleon interactions into an overall attractive nuclear and repulsive Coulomb potential felt by the two nuclei as a whole. The double folding model is one approach to determine the nucleusnucleus potential [1-3], the Time-Dependent HartreeFock (TDHF) approach is another $[4,5]$. In practice a simple Woods-Saxon parameterization is often used, with the

\footnotetext{
a e-mail: david.hinde@anu.edu.au

bermanent address: Nuclear Physics Division, Bhabha Atomic Research Centre, Trombay, Mumbai-400 085, India
}

potential diffuseness at the barrier radius being a key parameter, along with the barrier energy itself, arising from the sum of Coulomb and nuclear potentials. The centrifugal potential - an effective potential arising from conservation of angular momentum - is also important. The overall nucleus-nucleus interaction potential and barrier energy is obtained from the sum of these three terms, for any relative angular momentum $\mathrm{J} \hbar$.

If the collision energy is sufficient to allow the system to penetrate inside the barrier, fusion is assumed to occur. Fig. 1 illustrates the concept, with the radial separation being the key variable. Fusion and scattering are complementary outcomes arising from this physical picture. Fusion is implicitly treated as an irreversible process, removing flux (or probability) from the less complex scattering/reaction processes where the projectile-like and target-like nuclei remain identifiable. In practice this is achieved by a "mathematical trick", either an imaginary potential or an ingoing wave boundary condition applied well inside the fusion barrier radius. The compound nucleus hypothesis [6] of Bohr (illustrated qualitatively in Fig. 2) pictures irreversibility as actually arising from the sharing of the energy of the projectile amongst the nucleons of the combined system - equivalent to coupling to a (small but hot) heat bath.

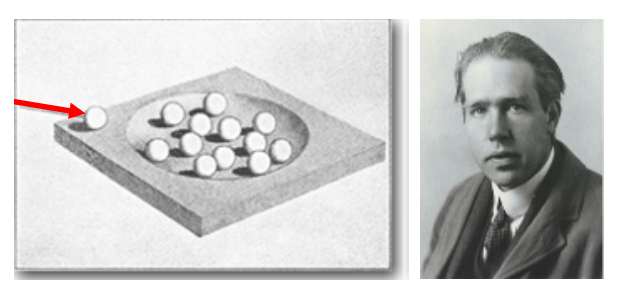

Figure 2. Compound nucleus formation (left) according to the picture of Bohr (right).

The flux that does not fuse in practice is treated within two very different frameworks. Outcomes where the collision partners emerge in a superposition of a limited number of discrete long-lived quantum states are described 

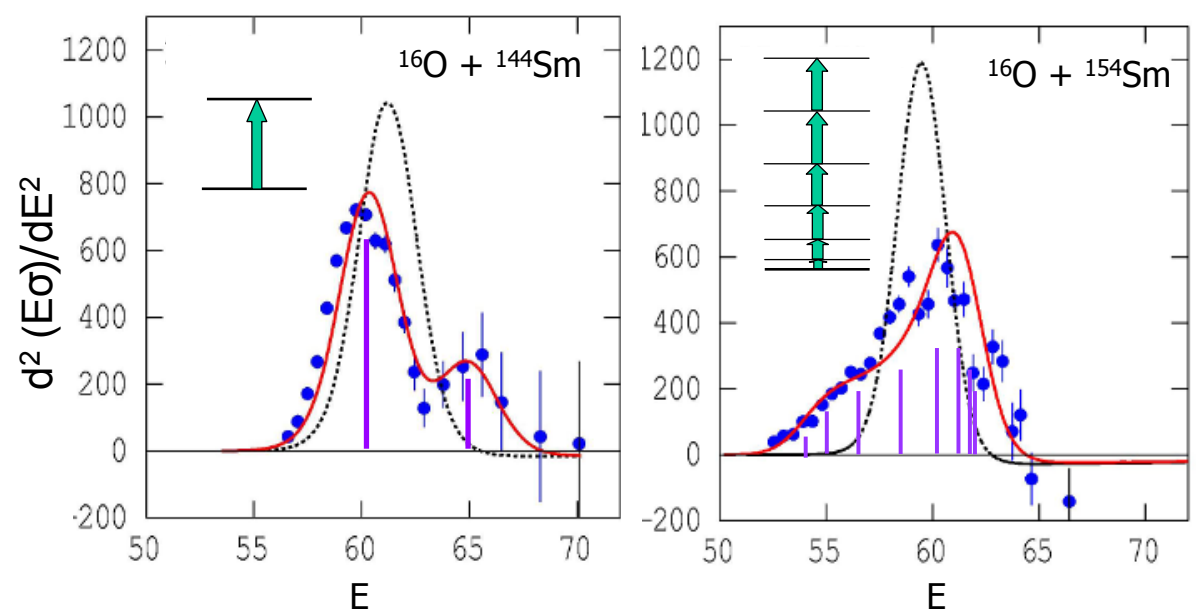

Figure 3. Experimental fusion barrier distributions (points) vs. centre-of-mass energy E. The discrete barriers (shown schematically in purple) within the eigenchannel approximation are shown for vibrational (left) and rotational (right) coupling schemes. The red line shows the calculated barrier distribution, which can be associated with the sum of those from each eigenbarrier, smoothed by quantum tunnelling. The calculated uncoupled distributions, also including tunnelling, are shown by the black dotted curves.

by the coupled channels model [7], which is based on the time-independent Schrödinger equation. The coupling matrix elements between pairs of channels are symmetric with respect to interchange of indices, meaning all transitions are reversible in the interaction region. However, in deep inelastic collisions (DIC - known as "the energy loss mode" in heavy-ion collisions) where high excitation energies and thus regions of high level density are populated, excitation energy is assumed to be irreversibly dissipated at the time of the collision in the same way as in fusion. DIC have been treated fully classically [8], semiclassically, using classical trajectories, using the GRAZING code, whose framework is described in Ref. [9], and in the quantum mean field TDHF theory [10].

The coupled-channels (CC) model has been enormously successful, providing the most comprehensive explanation of elastic and inelastic scattering and transfer to discrete states [11]. Channel couplings turned out also to have major effects on fusion, as predicted [12], explaining the experimentally observed phenomenon of enhancement of fusion cross-sections (by up to a factor of 1000) at energies below the average fusion barrier [13]. The concept of the experimental fusion barrier distribution [14], including (within some approximations) the concept of eigenbarriers, and its experimental realization [15-18] (examples are shown in Fig. 3) provided a new test of the coupledchannels model which, after refinement [19, 20], often succeeded in describing the major features observed. This success strengthened the belief that the coupled-channels model offers the best approach to describe near-barrier reactions. Thus a wide range of experimental evidence and theoretical interpretations indicate that the colliding nuclei are indeed in a coherent superposition of the quantum states of the separated nuclei as they approach the fusion barrier. Note that the standard $\mathrm{CC}$ model should not be able to describe DIC, where kinetic energy dissipation is understood to show a time-dependent increase related to the time the two nuclei remain in contact.

\section{Fusion excitation functions}

In measurements of fusion barrier distributions, the development of instrumentation to enable precise absolute cross section measurements has played an important role. Heavy fusion-evaporation residues (ERs) are the main product of nuclear fusion in reactions forming nuclei with atomic number $\mathrm{Z}<82$. Measuring ER cross sections requires understanding their kinematic characteristics. $\mathrm{Nu}-$ clear fusion products initially have the beam momentum. They are deflected from the beam direction by the small recoil momentum imparted by light particles evaporated during cooling to form evaporation residues. ERs are mostly found inside a $10^{\circ}$ cone centred around $0^{\circ}$. To measure fusion cross sections, the goal is to physically separate the ERs from the beam particles before detection, as the intensity of the Rutherford-scattered beam is at best $10^{4}$ times that of the ERs, and can be more than $10^{12}$ times larger.

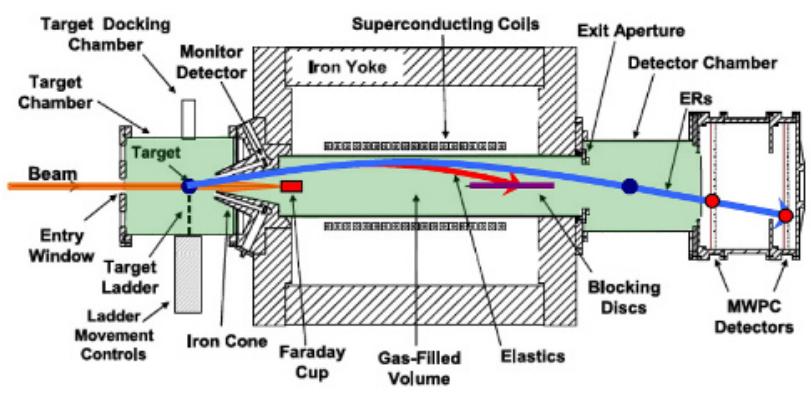

Figure 4. Schematic diagram of the components of the solenoidal separator SOLITAIRE. The He gas region is shown by the light green shading. The points where the ER trajectory is closest to the axis are shown by blue circles, and the interaction points with the two MWPC tracking detectors by red circles. The helical trajectories of elastically scattered beam particles (red) and ERs (blue) are represented by the radial separation from the axis. More details can be found in Ref. [21]. 
At the Heavy Ion Accelerator Facility in the Department of Nuclear Physics at the ANU, we first developed a small solid angle velocity filter [15] for this purpose. This has more recently been supplanted by the 6.5 Tesla superconducting solenoidal separator SOLITAIRE [21] (shown schematically in Fig.4), with a solid angle of $86 \mathrm{msr}$. In a solenoidal field, in simple terms, the radial component of the particle velocity interacts with the axial component of the field, bending trajectories back to the beam (solenoid) axis at different focal lengths, depending on the ratio of momentum to charge state. With a gas pressure such that there are a sufficient number of chargechanging collisions, particle trajectories will correspond to those for the mean charge state in the gas. In He gas the ERs have a lower charge state than the beam particles; He gas gives optimal separation between ERs and scattered beam. Detailed ER cross section excitation functions have been measured for fusion of ${ }^{58} \mathrm{Ni}$ with ${ }^{58,60,64} \mathrm{Ni}$, allowing high quality fusion barrier distributions [13] to be determined [21], as shown in Fig. 5. The newer data demonstrate good agreement with the results of Ref. [17], confirming the strong role of multiphonon excitations in both nuclei. Recently, the barrier distribution has been measured for fusion of ${ }^{46} \mathrm{Ti}+{ }^{124} \mathrm{Sn}$ to investigate the role of transfer [22].

The solenoid is also at the core of the development of a radioactive beam capability at the ANU [23-25], using the solenoid-based in-flight transfer method pioneered at Notre Dame University [26, 27]. The latest developments are described in the contribution of I.P. Carter et al. to these proceedings.

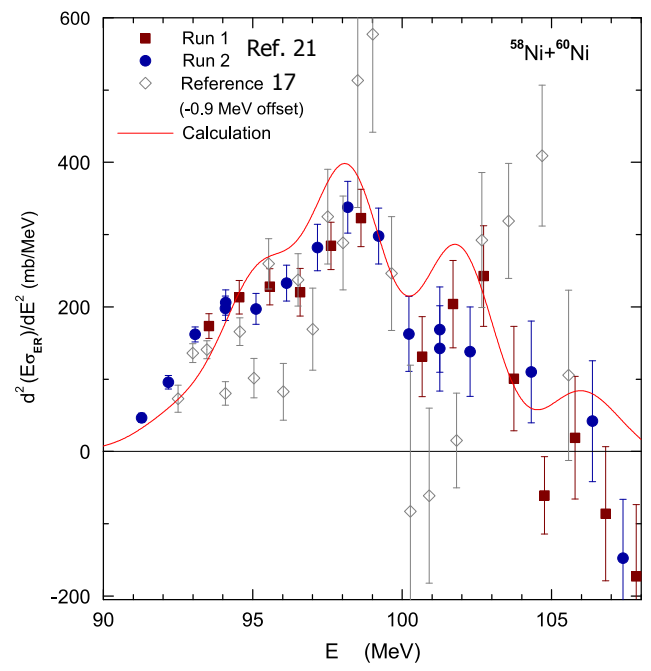

Figure 5. Fusion barrier distributions for the reaction ${ }^{58} \mathrm{Ni}+{ }^{60} \mathrm{Ni}$ as a function of centre-of-mass energy. The faint points are from the work of Ref. [17], the filled points from two separate excitation function measurements using SOLITAIRE [21]. The red curve is a coupled-channels calculation with three quadrupole phonon excitations in both nuclei.

Despite the successes of the coupled-channels model in reproducing the major features of many fusion barrier distributions, some aspects of fusion have not been satisfactorily described by the model. The first problem is
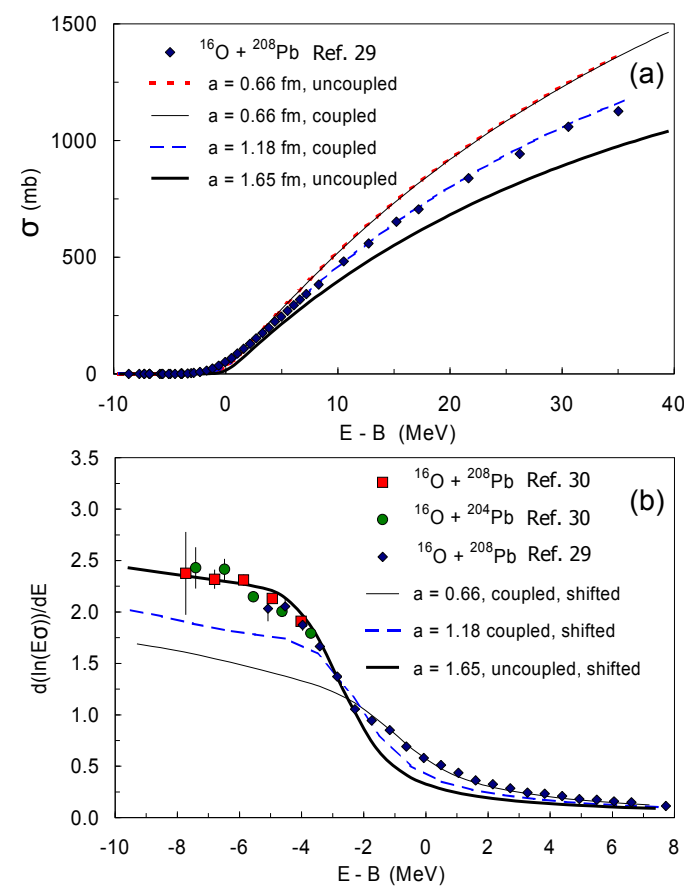

Figure 6. Fusion cross section data with respect to the average fusion barrier energy B. Data are from Refs. [29, 30]. A calculation with standard diffuseness $a=0.66 \mathrm{fm}$ fails to match the measurements. Using a larger value of $a$ reproduces $\sigma$ above the barrier (a), but fails to reproduce the low energy logarithmic slope (b), and vice-versa (see Ref. [30]).

the observation of fusion cross sections lower than predicted, both at above-barrier and at deep sub-barrier energies. The second issue is the reduction of complete fusion for reactions of weakly bound nuclei. The third aspect is the massive suppression of fusion by quasifission in reactions forming very heavy nuclei. These are all related to important physical processes beyond the original coupledchannels framework. Each will be described in more detail in the following sections.

\section{Inhibition of quantum tunnelling}

Fusion forming heavy elements with $\mathrm{Z}>82$ leads predominantly to fission, which is measured at the ANU with high efficiency using the CUBE spectrometer [28]. This currently consists of two large area MWPC detectors, each subtending $75^{\circ}$ in scattering angle. With these detectors, many fission cross section excitation functions have been measured. It must be noted here that measured fission characteristics (see Section 7) show that after capture in the potential pocket, true fusion - forming a compact compound nucleus - does not necessarily occur; thus, passage inside the entrance-channel potential pocket will be referred to as capture subsequently, which includes both fusion and quasifission.

The capture (fission + ER) excitation function for the ${ }^{16} \mathrm{O}+{ }^{208} \mathrm{~Pb}$ reaction has been precisely measured at ANU [30], with cross sections covering eight orders of magnitude. These data showed clearly that standard CC 
calculations, based on the barrier-passing picture, could not simultaneously describe deep sub-barrier and abovebarrier data with a single potential. This is illustrated in Fig. 6, where a standard Woods-Saxon nuclear potential diffuseness $(a=0.66 \mathrm{fm})$ under-predicts the measurements both (a) in the above-barrier region and (b) below-barrier (where the logarithmic slope is presented). No potential shape (diffuseness) reproduces both regimes simultaneously.

The question thus arises, what Woods-Saxon diffuseness value best represents the nuclear potential shape around the barrier radius? This has been re-examined experimentally in recent years, following the suggestion of Ref. [31]. There it was proposed that high precision quasielastic scattering data at sub-barrier energies should unambiguously reveal the diffuseness. This can be understood in a classical picture, since the tail of the nuclear potential perturbs the trajectories, which results in a slight suppression of yield at the more backward angles. New measurements with uncertainties approaching $0.1 \%$ were made, and analysis carried out $[32,33]$ within the coupledchannels framework. A quantum interference approach was also developed to address the same question [34], including collisions of identical nuclei [35]. All showed results essentially independent of the channel couplings, and consistent with a diffuseness parameter $a$ between 0.6 and $0.7 \mathrm{fm}$. Thus it was concluded that the large diffuseness required to fit fusion cross sections, as well as the inconsistency between above-barrier and below-barrier fusion, was truly reflecting physical effects not included in the CC model.

A careful systematic analysis of many above-barrier fusion cross section excitation functions was carried out $[36,37]$. It showed that as the charge product of the reaction increases, fusion is increasingly suppressed compared to calculations using the standard nuclear potential diffuseness (confirmed to be appropriate from the above scattering analysis). It was suggested [37] that deep-

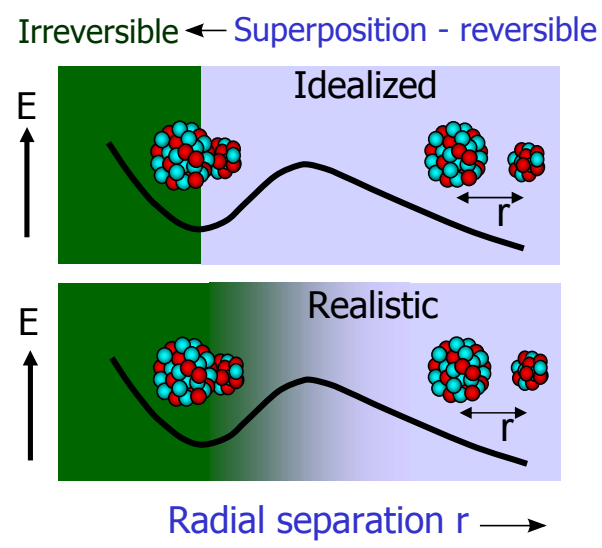

Figure 7. The idealized coupled-channels picture of nuclear collisions is illustrated above. The light blue represents the coherent superposition, the dark green a sudden onset of irreversible energy dissipation. A more realistic picture is sketched below. The questions are, at what radial separation does irreversible energy dissipation play a role, and what is the mechanism? inelastic scattering may be responsible for the suppression. Although DIC are considered to be predominantly a phenomenon associated with high angular momenta, experimental evidence existed for DIC at near-barrier energies [38].

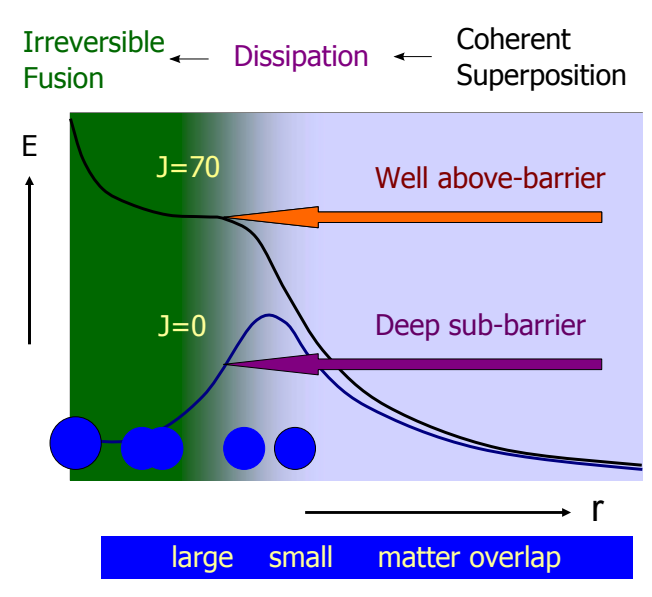

Figure 8. It is seen qualitatively that the matter overlap (indicated on the lowest scale) at a high energy and angular momentum is the same as at the inner turning point at a matching energy below the barrier. Assuming that the probability of energy dissipation depends primarily on the matter overlap, energy dissipation can also be expected to play a role in sub-barrier fusion (by quantum tunnelling) if it is important in above-barrier fusion.

Later it was suggested $[39,40]$ that since the transition from coherent superposition to irreversible energy dissipation must occur gradually, it could affect more aspects of capture (including quantum tunnelling [40]) if its onset were close to the barrier radius rather than far inside. This idea is sketched in Fig. 7. The shaded band around the potential represents the barrier distribution arising from couplings. Based on this idea, it was proposed [30] that one contributor to the inhibition of fusion seen for the ${ }^{16} \mathrm{O}+{ }^{208} \mathrm{~Pb}$ reaction, both below and above the barrier, could be the critical assumption of the coupled-channels model that the system remains in a coherent superposition of states all the way into the potential pocket. In practice these states comprise the limited subspace of states (generally ground-state and low energy collective states of the projectile and target nuclei) included in the CC calculations. The questions that arise from this are, at what radial separation does irreversible energy dissipation play a role, and what is the mechanism? Independent of its exact nature, additional physical processes beyond the $\mathrm{CC}$ framework would be expected to depend primarily on the matter overlap. Through this idea, effects on fusion at both high and low beam energies can be related to each other. This relationship is illustrated qualitatively in Fig.8, where the same matter overlap is encountered at the outer turning point for a high angular momentum as at the inner turning point at low angular momentum. Thus if energy dissipation is important at the limiting angular momenta at energies well-above the barrier, it should play a role at deep sub-barrier energies also. A key question then is whether 


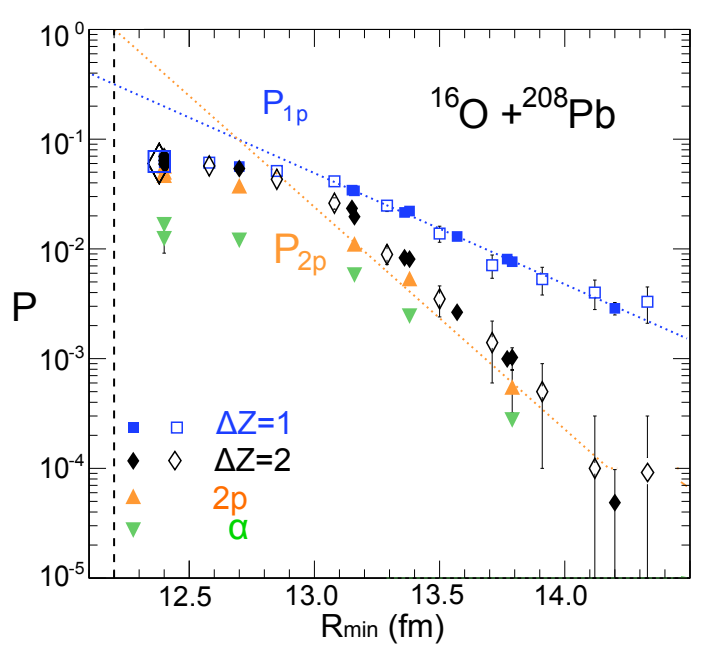

Figure 9. Transfer probabilities $P$ as a function of the distance of closest approach for one and two proton and alpha stripping reactions, from earlier work (hollow points) and from Ref. [41]. The dotted lines represent exponential fits to the data beyond 13 $\mathrm{fm}$. The vertical dashed line shows the fusion barrier radius.

there is evidence for energy dissipation in below-barrier collisions.

\section{Energy dissipation below-barrier}

Evidence for DIC at near-barrier energies has been observed [42] for the reaction ${ }^{40} \mathrm{Ca}+{ }^{208} \mathrm{~Pb}$ at energies $5 \%$ above the barrier. It was also inferred from measurements for reactions of ${ }^{58} \mathrm{Ni}$ and ${ }^{32} \mathrm{~S}$, even at energies $5 \%$ or more below the barrier $[38,43]$. However, is there any evidence for outcomes analagous to DIC in sub-barrier reactions with a ${ }^{16} \mathrm{O}$ projectile?

ANU experimental quasi-elastic scattering data [32, 33] showed substantial loss of kinetic energy (TKEL) even at sub-barrier energies. With particle identification [41], the probabilities of each transfer mass partition, and their excitation energy spectra, were extracted as a function of the distance of closest approach by measuring back-angle excitation functions. The dependence of the total transfer probability on radial separation (Fig. 9) shows a steep exponential rise as the barrier radius is approached. Despite the absorption by fusion near the barrier, probabilities of $\sim 10 \%$ were found for transfer reactions with both $\Delta \mathrm{Z}=1$ and 2. Furthermore, the energy spectra following transfer showed population to excitation energies of $10 \mathrm{MeV}$ for sub-barrier ${ }^{16} \mathrm{O}+{ }^{208} \mathrm{~Pb}$ reactions (see Fig. 10). As shown for $E / V_{B}=0.96$, the population at high $\mathrm{E}_{x}$ is small, but it rises rapidly as the separation decreases, with a nonnegligible probability when extrapolating to the barrier radius (vertical dashed line in Fig. 9) or even inside. This suggests that the picture shown in Fig. 7 is realistic even for this low charge product reaction.

For ${ }^{32} \mathrm{~S}$ and ${ }^{40} \mathrm{Ca}$ reactions, excitation energies extend to $\sim 20 \mathrm{MeV}$ and $30 \mathrm{MeV}$ respectively (the latter from a preliminary analysis of a collaborative experiment at LNL). The excitation energy spectra for the $2 p$ stripping channel at sub-barrier energies $\left(\mathrm{E} / \mathrm{V}_{B}=0.96\right)$ are shown in Fig. 10. At least that part of the excitation energy spectrum above $8 \mathrm{MeV}$ might reasonably be associated with irreversible energy dissipation, in analogy with Bohr's picture for fusion with a neutron. The figure shows that the fraction of the transfer yield leading to such excitation energies (magenta shading) increases with increasing charge product. This is consistent with the conclusions of the empirical analysis [37] of above-barrier fusion suppression.

Can it be shown that missing kinetic energy is indeed transformed into excitation energy, and not (for example) carried away by a promptly emitted light particle? In reactions with actinide nuclei, the answer is yes. Excitation of heavy (target-like) nuclei to energies above their fission barriers can result in fission. ANU measurements [44] have shown evidence (illustrated in Fig. 11) for substantial fission cross sections following transfer reactions at sub-barrier energies. These measurements confirm that thermal excitation of the heavy target-like nucleus occurs, and with significant probability - our most recent data for ${ }^{40} \mathrm{Ca}+{ }^{238} \mathrm{U}$ suggest that such cross sections can be larger than those for fusion, even at above-barrier energies. Semi-classical calculations with the code GRAZING at above-barrier energies also indicate that competition between DIC and fusion is important to get a complete picture [45].

Standard CC models describing the coherent quantum superposition vital to describe scattering and capture are not able to describe the deep inelastic processes. A model framework should be developed to simultaneously include both CC effects and the dissipation that results in DIC. Steps are being taken in that direction [46]. Microscopic approaches such as TDHF should also be considered. Such calculations are able to reproduce sequential transfer [47, 48] and correlated pair transfer [49], and could be used to investigate the excitation energy of the fragments.

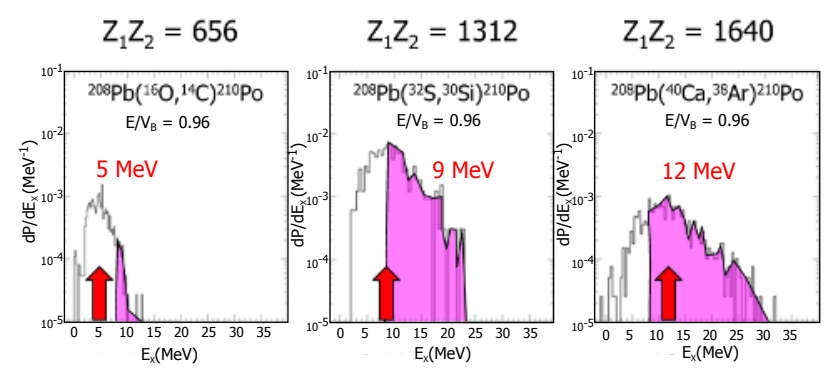

Figure 10. Excitation energy spectra for $2 p$ stripping reactions of ${ }^{16} \mathrm{O},{ }^{32} \mathrm{~S}$ and ${ }^{40} \mathrm{Ca}$ with ${ }^{208} \mathrm{~Pb}$ at the same sub-barrier energies $\mathrm{E} / \mathrm{V}_{b}=0.96$. The average excitation energy is indicated in red, whilst the magenta shading highlights excitation energies above $8 \mathrm{MeV}$. 


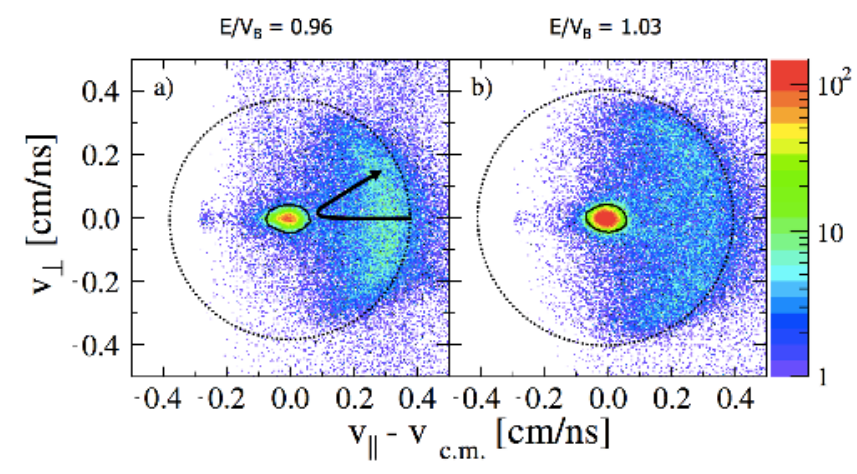

Figure 11. Fission source velocity components parallel and perpendicular to the beam axis, for fission events observed in the ${ }^{32} \mathrm{~S}+{ }^{232} \mathrm{Th}$ reaction, both below and above the barrier. Two-body events are in the centre of the plots, whilst the dashed circle shows the expected locus of heavy recoil velocities following elastic scattering. The events lying inside this circle correspond to the projectile losing momentum, and sufficient energy simultaneously being dissipated in the target-like nucleus to cause fission. This demonstrates energy dissipation triggered by transfer reactions below the fusion barrier.

\section{Suppression of complete fusion for weakly bound nuclei}

The coupled-channels picture has emerged from studies of well bound nuclei. For such nuclei the lifetimes of the strongly coupled collective states are very much longer than nuclear collision times of $\sim 10^{-22} \mathrm{sec}$. How do the reaction dynamics change when lifetimes of strongly coupled states are similar to or less than collision times? Answering this is topical due to the increasing opportunities worldwide to perform experiments with unstable nuclei.

More than a decade ago the first measurements were carried out showing unambiguously [50-52] that in reactions of weakly bound stable nuclei, complete fusion cross-sections at above-barrier energies are suppressed to $\sim 70 \%$ of those expected for well-bound nuclei. This conclusion relied on measurements of unprecedented precision and completeness for such reactions, as well as the application of the experimental fusion barrier distribution concept. Similar or identical suppression of complete fusion has since been inferred in many other reactions with weakly bound stable nuclei. It was natural to assume that the suppression of complete fusion is caused by the direct breakup of the weakly bound nuclei into their cluster constituents, e.g. ${ }^{6} \mathrm{Li}$ breaking into ${ }^{4} \mathrm{He}$ and ${ }^{2} \mathrm{H}$. Some measurements appeared to support this idea, but many did not. It thus remained a perplexing question.

Experiments measuring breakup itself can clarify the situation in a model-independent way, if all the breakup fragments are detected, and none can be captured by the other (target) nucleus. A 512 pixel detector array at backward angles was therefore developed $[53,54]$ to measure all breakup processes in reactions of ${ }^{6,7} \mathrm{Li}$ and ${ }^{9} \mathrm{Be}$. Energies below the barrier were chosen to minimize fragment absorption that complicates interpretation. The angles of the fragments and their measured energies are used to determine the energy of the recoiling target-like nucleus, and

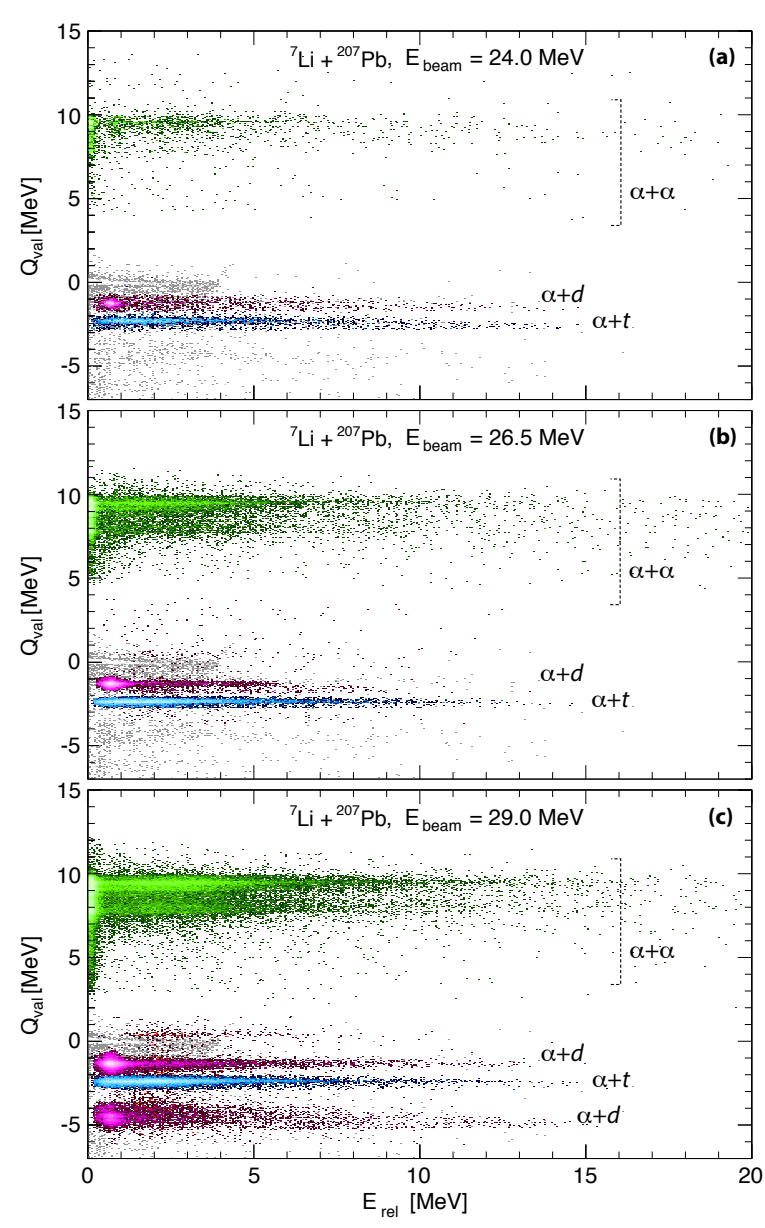

Figure 12. The reaction $\mathrm{Q}$-value plotted against the relative energy of the two fragments $\left(E_{r e l}\right)$ provides a complete picture of the breakup dynamics. Measurements are shown for belowbarrier ${ }^{7} \mathrm{Li}$ beam energies from 24 to $29 \mathrm{MeV}$ on a ${ }^{207} \mathrm{~Pb}$ target. Coincidence pairs, $\alpha+d, \alpha+t$, and $\alpha+\alpha$ (identified by different colours) result from breakup following $n$-stripping, inelastic excitations and $p$-pickup, respectively. Intensity is shown by the colour saturation, where pale colours indicate high intensity. The measured $E_{r e l}$ provides information on the state of the projectilelike nucleus prior to breakup and on breakup time-scales.

thus the reaction $Q$-value, through momentum conservation assuming three-body kinematics. The $Q$-value spectra for breakup events show sharp peaks, demonstrating that the breakup itself is indeed almost exclusively binary, validating the application of three-body kinematics. Knowledge of the $Q$-values provides information about the breakup mode, and states populated in the target-like nucleus, but not about the states of the projectile-like nucleus. However, at breakup, the kinetic energy available to share between the breakup fragments is the sum of the breakup $Q$-value and the excitation energy of the state from which breakup occurs. Information on the state of the projectile-like nucleus prior to breakup is thus contained in the relative energy $\left(E_{r e l}\right)$ of the two breakup fragments.

The reaction $Q$-value together with $E_{r e l}$ gives the most complete characterization available of the various breakup processes. Fig. 12 shows plots of $Q$-value against $E_{r e l}$ for 
reactions of ${ }^{7} \mathrm{Li}$ with ${ }^{207} \mathrm{~Pb}$ at three sub-barrier energies. The $\alpha+t$ pairs result from breakup of ${ }^{7} \mathrm{Li}$; this observation matches the expectation that ${ }^{7} \mathrm{Li}$ breaks up into its cluster constituents. However, several other breakup modes are prominent. The $\alpha+\alpha$ pairs originate from breakup of the ${ }^{8} \mathrm{Be}$ nucleus which is formed when ${ }^{7} \mathrm{Li}$ picks up a proton from the closed proton-shell nucleus ${ }^{207} \mathrm{~Pb}$. The $\alpha+d$ pairs are due to the breakup of ${ }^{6} \mathrm{Li}$ formed in $n$-stripping reactions. The different mechanisms change their relative yields as a function of beam energy, due to the different range of the triggering mechanisms (Coulomb, or neutron and proton transfer).

The narrow peaks at low $E_{r e l}$ (e.g. $\alpha+\alpha$ with $E_{r e l}=$ $92 \mathrm{keV}$ from ${ }^{8} \mathrm{Be}$ ground-state decay) are associated with breakup on the outgoing trajectory, far from the target nucleus, whose Coulomb field thus cannot introduce a significant perturbation to the observed $E_{r e l}$. Such long-lived breakup mechanisms cannot affect fusion at above-barrier energies, as the fusion process is much faster. In contrast to the narrow peaks, a broad distribution at high $E_{r e l}$ is calculated $[55,56]$ for breakup on the ingoing trajectory (i.e. prompt breakup). This can result in suppression of complete fusion at above-barrier energies. An analogy is shining two parallel light beams at a spherical mirror - on reflection, the beams diverge. The largest contribution to prompt breakup, having large $E_{\text {rel }}$, is from $\alpha-\alpha$ breakup following $p$-pickup [54]. More details are provided in the contribution of D.H. Luong et al. in these proceedings.

Our conclusion is that prompt breakup of weaklybound stable nuclei can be triggered by different processes: predominantly $n$-stripping for ${ }^{6} \mathrm{Li}$ and ${ }^{9} \mathrm{Be}$ [53], but $p$-pickup for ${ }^{7} \mathrm{Li}$. This means that the probability and characteristics of breakup, and its influence on fusion, depend not only on the properties of the initial nucleus, but also on those of its neighbours. Crucially, the measurements reveal those mechanisms that lead to breakup that is fast enough $\left(\sim 10^{-22} \mathrm{~s}\right)$ to affect fusion. These results will provide important input to developing a complete model of reactions involving both $\alpha$-cluster nuclei and exotic nuclei at the limits of existence (becoming available from radioactive isotope accelerators), with potential applications in nuclear astrophysics (see contribution to this conference by K.J. Cook et al.).

\section{Fusion suppression by quasifission}

Fusion leading to the formation of very heavy elements may be conceptually divided into two stages. The first is when the two nuclear surfaces come into contact, associated with capture in the entrance-channel potential pocket. Then the elongated dinucleus formed at contact explores the potential energy surface (PES), to find the compact compound nucleus $(\mathrm{CN})$ shape at the potential minimum. This is the end-product of the fusion process. If the $\mathrm{CN}$ survives the subsequent statistical fission decay (through particle evaporation to an excitation energy below the fission threshold) it eventually forms the desired (super)heavy evaporation residue. The cross section for heavy element formation via fusion-evaporation is given by

$$
\sigma_{E R}=\sum_{J=0}^{\infty} \sigma_{J}\left(E_{c . m .}, J\right) \cdot P_{C N}\left(E^{*}, J\right) \cdot W_{s u r}\left(E^{*}, J\right),
$$

where $\sigma_{J}\left(E_{c . m .}, J\right)$ is the capture cross section as a function of center-of-mass energy $E_{c . m}$. and angular momentum $J \hbar$, $P_{C N}\left(E^{*}, J\right)$ is the probability that the system will reach the compact configuration as a function of the excitation energy $E^{*}$ and $J$, and $W_{\text {sur }}\left(E^{*}, J\right)$ is the probability that the system will survive statistical fission decay through sequential particle evaporation.

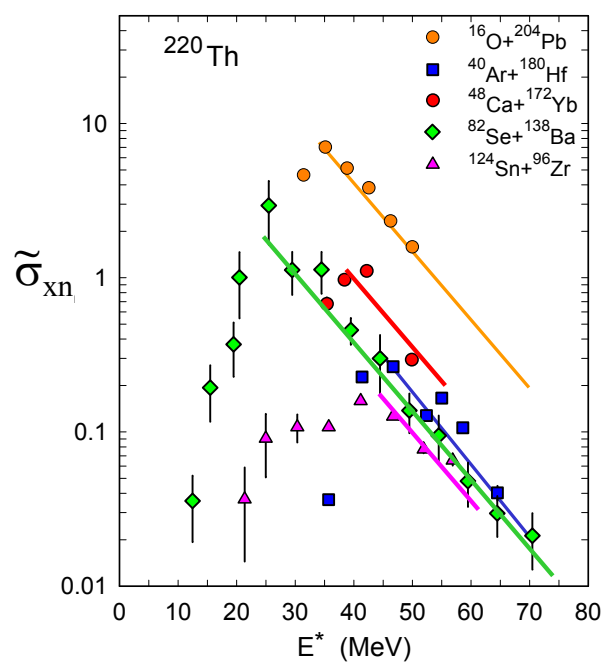

Figure 13. Total xn ER cross sections divided by $\pi t^{2}$ as a function of excitation energy, for reactions forming ${ }^{220} \mathrm{Th}$ compound nuclei. Trends for the same projectile-target combination are highlighted by the lines. The ${ }^{16} \mathrm{O}$ induced reaction gives yields typically 10 times those of reactions with heavier projectiles.

For fusion reactions forming very heavy elements, after capture many systems fail to reach the compact $\mathrm{CN}$ shape, instead undergoing fission from a more elongated shape, resulting in $\mathrm{P}_{\mathrm{CN}}<1$. This process, competing with formation of a compact $\mathrm{CN}$, is called quasi-fission (QF) [57-59].

The surest indication of formation of a compact $\mathrm{CN}$ is the observation of ERs, because it is often difficult to separate fusion-fission from quasi-fission (since their characteristics often have considerable overlap). At abovebarrier energies, where the angular momenta leading to ER formation are fully populated in the capture process, $W_{\text {sur }}\left(E^{*}, J\right)$ is the same for each reaction [60], thus ratios of cross sections (scaled by the different reduced wavelengths $\lambda$ to give $\tilde{\sigma}$ ) for different reactions provide relative values of $\mathrm{P}_{\mathrm{CN}}[60]$.

Experimental results [61] are shown in Fig. 13 for reactions forming ${ }^{220} \mathrm{Th}$. Here $\tilde{\sigma}_{\mathrm{xn}}$ is presented as a function of the ${ }^{220} \mathrm{Th}$ excitation energy. It was a surprise that the yields for the ${ }^{16} \mathrm{O}$-induced reaction are typically a factor of 10 above those for the heavier projectiles. The yields amongst the latter show a much smaller change with mass-asymmetry. These facts have been interpreted $[60,61]$ as indicating the critical importance of the 

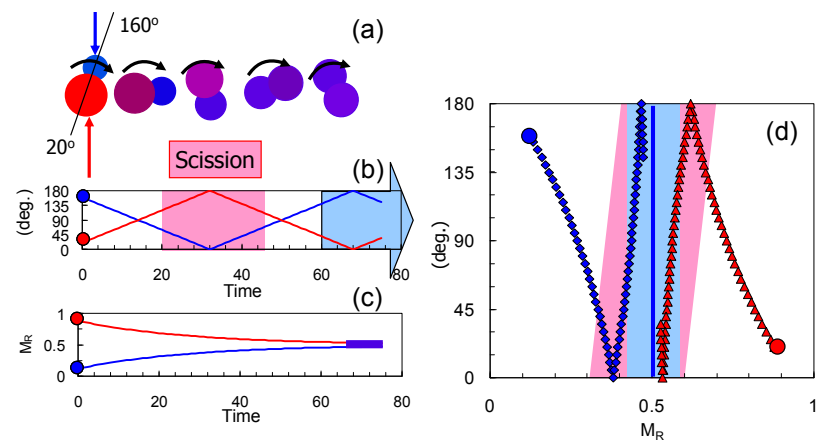

Figure 14. The relationship between sticking time and the massangle distribution. For the reaction sequence sketched in (a), the time dependence (arbitrary units) of angle (b) and mass-ratio (c) are shown. Such short times cannot be measured directly, but angle and mass-ratio can. The individual mass and angle dependencies combine to give a trajectory (d) on the MAD for a single impact parameter. Including a range of impact parameters, scission after half a turn (pink) or a full turn (blue) will give strong or weak mass-angle correlations respectively.

entrance channel mass-asymmetry compared to the position of the Businaro-Gallone peak. Thus according to this argument, among the reactions forming ${ }^{220} \mathrm{Th}$, a key reason that the ${ }^{16} \mathrm{O}$-induced reaction has the highest $\mathrm{P}_{\mathrm{CN}}$ is because the small projectile tends to be absorbed ("eaten up") by the larger target nucleus, unlike the more masssymmetric reactions, which tend to move towards masssymmetry.

\section{Quasifission dynamics through mass-angle distributions}

As a wholly dynamical process, a key quantity characterizing quasifission is its timescale - that is, the "sticking time" between capture and breakup (scission). The measurement of the full range of mass-splits between projectile and target over a wide range of scattering angles results in a two-dimensional matrix - the mass-angle distribution (MAD). Measurements of quasifission MADs at GSI in the 1980s [59,62] showed that QF timescales could often be shorter than the rotation time of $\sim 10^{-20} \mathrm{~s}$, and were thus significantly shorter than the typical timescale of fusionfission. The measurement of MADs thus offers a key insight into quasifission dynamics.

\subsection{Principle behind the mass-angle distribution}

The relationship of the MAD to the "sticking time" between capture and scission is illustrated schematically in Fig. 14. The projectile nucleus (blue) is incident from the top of the page, and sticks to the larger target nucleus (red). The system then rotates, Fig. 14(b) illustrating schematically angle against time (in arbitrary units) for a single angular momentum value; in a real reaction a distribution will be present. For a parabolic potential, mass-symmetry is approached with an expected time dependence 1 - $\exp \left(-t / \tau_{e q}\right)$, where $\tau_{e q}$ is the mass-equilibration time constant [59]. This dependence is sketched in Fig. 14(c).

If scission happens soon after initial contact, then little mass change can occur, and a projectile-like fragment is ejected with mass-ratio $M_{R}$ (defined as the mass of one fragment divided by the total mass) at a backward angle $\theta_{c . m .}$. The complementary partner with mass-ratio $\left(1-M_{R}\right)$ is found at $\left(\pi-\theta_{c . m .}\right)$. A longer sticking time of the system results in larger rotation angles, and also allows more mass exchange. This evolution is illustrated on the MAD shown in Fig. 14(d). Rotation of the system by $\sim 180^{\circ}$ still results in a substantial mass-angle correlation (pale pink shading in Fig. 14(b),(d)), whilst once the system has turned $\sim 360^{\circ}$ or more, the correlation between the mass ratio and fragment emission angle is washed out, resulting in symmetric mass splits on average, independent of angle (blue shading).

\subsection{Effect of deformation alignment on quasifission}

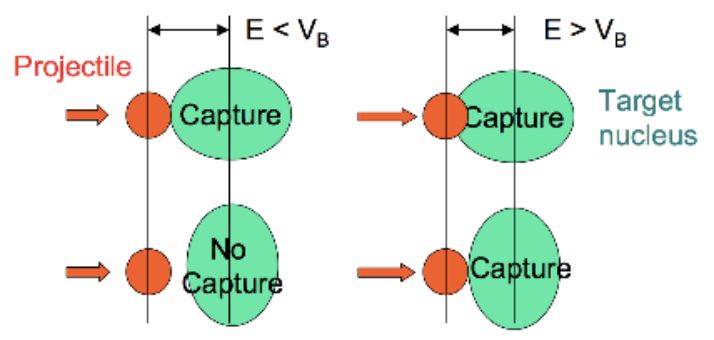

Figure 16. Diagram illustrating the effective deformation alignment of statically deformed nuclei at sub-barrier energies (left panel), and the averaging over all orientations at above-barrier energies (right panel). The black arrows represent the different distances of closest approach at each energy.

For nuclear collisions involving prolate nuclei (e.g. ${ }^{232} \mathrm{Th},{ }^{238} \mathrm{U}$ ), when the deformation axis is aligned with the projectile nucleus, the dinucleus is very elongated at contact (Fig. 16 upper images), whereas it is more compact if anti-aligned (lower images). With decreasing c.m. energy $\mathrm{E}$, increasing deformation alignment occurs, because of the correlation of the capture barrier energy with orientation [13, 18, 28, 63]. The physical reason for this effective deformation alignment at sub-barrier energies is sketched in Fig. 16. The consequences are seen in the MADs for ${ }^{32} \mathrm{~S}$ $+{ }^{232} \mathrm{Th}$, shown in Fig.15, which were measured at five energies, from below $\mathrm{V}_{B}(154.5 \mathrm{MeV})$ to above.

The MADs and the projections onto $\mathrm{M}_{R}$ in Fig. 15 show mass-asymmetric and mass-symmetric components, with the weight of each changing rapidly with $E / V_{B}$. The mass-asymmetric component is dominant below $V_{B}$. The MADs show that the dinuclear system rotates typically by $<90^{\circ}$ before scission (short arrows in Fig. 15). The more mass-symmetric component becomes dominant above $\mathrm{E} \sim \mathrm{V}_{B}$. It appears to be completely mass-symmetric in the $\mathrm{M}_{R}$ spectra - and could be taken to be consistent 


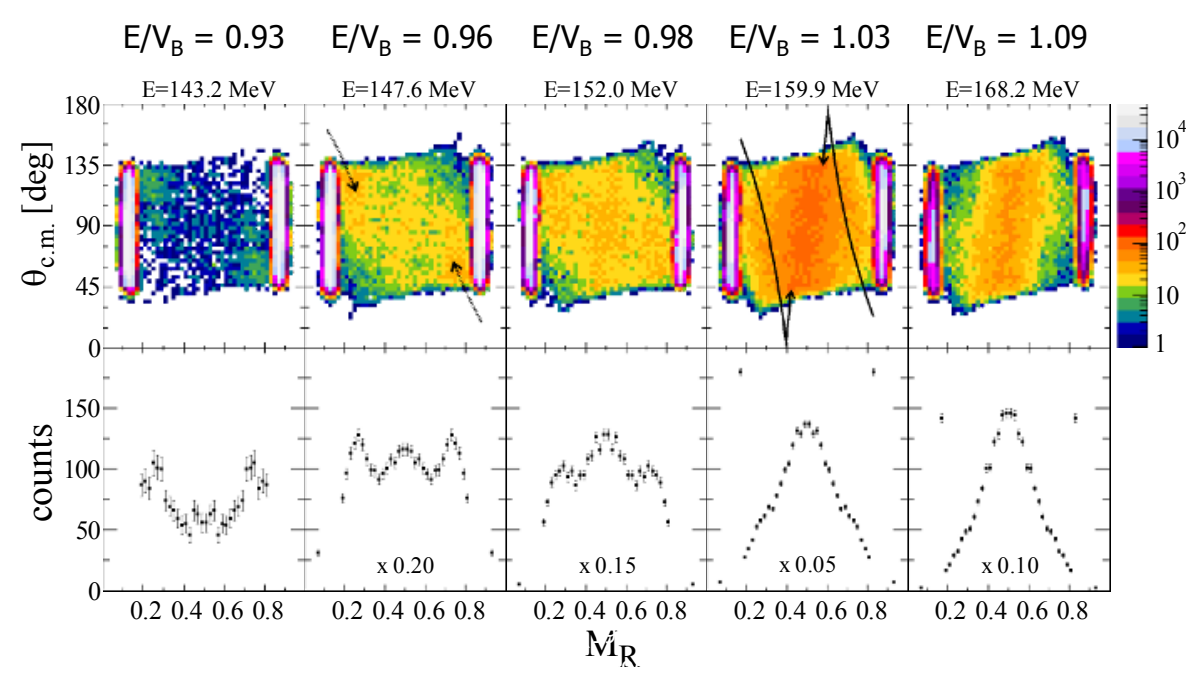

Figure 15. Mass-angle distributions of fission following capture for the reaction ${ }^{32} \mathrm{~S}+{ }^{232} \mathrm{Th}$. Centre-of-mass beam energies $\mathrm{E}$ (indicated) span the average capture barrier energy $\mathrm{V}_{B}$. The projected fission mass-ratio distributions (below) show a transition from dominantly mass-asymmetric at sub-barrier energies, to apparently mass-symmetric at $\mathrm{E}>\mathrm{V}_{B}$. In the MAD the "mass-symmetric" component shows a significant mass-angle correlation, inconsistent with fusion-fission.

with fusion-fission. In the MADs, this component has a significant mass-angle correlation, corresponding to rotation of the dinuclear system before scission by $\sim 180^{\circ}$ (long arrows). The correlation of mass with angle seen in the MADs at the higher energies shows that this "masssymmetric" component is inconsistent with fusion-fission, but rather appears to be dominated by quasi-fission. Fission angular distributions [64] confirmed this. These experimental mass distributions are consistent with measurements for ${ }^{238} U+{ }^{32} S[62]$, and ${ }^{36} S+{ }^{238} U$ [65], although the interpretations may differ.

\subsection{Effect of entrance channel closed shells}

To investigate the effect on quasifission of closed shells in the entrance channel, measurements [66] of MADs were made for ${ }^{40,44,48} \mathrm{Ca}$ projectiles bombarding thin targets of ${ }^{208,204} \mathrm{~Pb}$ (forming ${ }^{248,252} \mathrm{No}$ with $\mathrm{Z}_{C . N .}=102$ ), and for ${ }^{48} \mathrm{Ti}$ bombarding ${ }^{200} \mathrm{Hg}\left({ }^{248} \mathrm{No}\right)$ and ${ }^{208} \mathrm{~Pb}\left({ }^{256} \mathrm{Db}\right)$. Measurements were made a few percent below the average fusion barrier energy to ensure low relative velocity at contact. The MADs and projected $\mathrm{M}_{R}$ spectra are shown in Fig. 17, together with reference data for the ${ }^{16} \mathrm{O}+{ }^{238} \mathrm{U}$ reaction at an above-barrier energy, forming ${ }^{254} \mathrm{Fm}$.

The Gaussian fit standard deviations $\sigma_{M R}$, with experimental uncertainties, and the $\sigma_{M R}$ value for ${ }^{48} \mathrm{Ca}+{ }^{208} \mathrm{~Pb}$ from Ref. [67] are also shown in the table associated with Fig. 17. Despite having similar or identical $\mathrm{Z}_{1} \mathrm{Z}_{2}$, and forming similar or identical compound nuclei, there is a wide variation in the $\sigma_{M R}$ values, indicating a significant variation in the probability/characteristics of quasifission.

To help understand this, Fig. 18 shows $\sigma_{M R}$ as a function of the number of magic numbers $\mathrm{N}_{m}$ in the entrance channel. There is a strong correlation, with $\sigma_{M R}$ approaching the expectation for fusion-fission $\left(\sigma_{M R}=0.07\right)$ with increasing $\mathrm{N}_{m}$. This suggests that reactions involving nuclei having several magic numbers form a true compact compound nucleus with higher probability. It seems likely that this is associated with reduced energy dissipation as the two nuclei are overlapping, allowing more compact shapes to be reached. Supporting this hypothesis, a systematic analysis of $\mathrm{xn}$ evaporation residue cross sections in reactions forming Th isotopes has suggested some enhancement in fusion probabilities for reactions with two magic numbers in the entrance channel [68].

It is notable that the ${ }^{40} \mathrm{Ca}+{ }^{208} \mathrm{~Pb}$ reaction, which has maximal magicity, has a large $\sigma_{M R}$. Only this reaction has a large N/Z asymmetry. Protons and neutrons will tend to be transferred early in the reaction to equalize N/Z. TDHF calculations [66] of nucleon exchange prior to capture confirm this. Thus matching of $\mathrm{N} / \mathrm{Z}$ in the entrance channel should be an important condition in the enhancement of fusion in reactions of magic (and especially doubly-magic) nuclei.

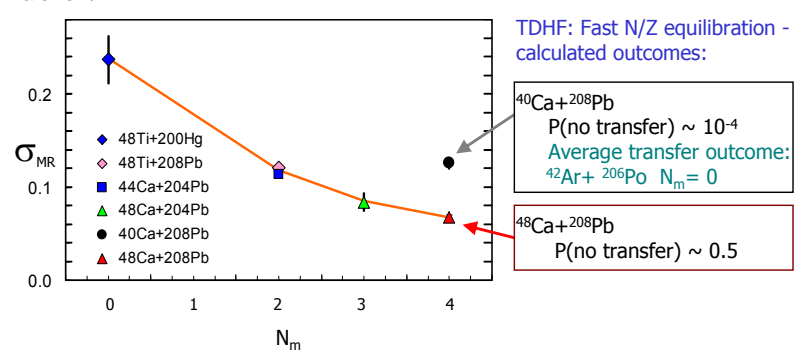

Figure 18. (Left) Standard deviations $\sigma_{M R}$ of the fission-like mass distributions as a function of the number $N_{m}$ of magic numbers in the entrance channel. The line guides the eye. The expectations of $\sigma_{M R}$ for fusion-fission are in the range 0.06 to 0.08. (Right) TDHF calculation outcomes are summarized (see Ref. [66]).

\subsection{Quasifission time scale}

From the theoretical point of view, quasifission times can be predicted using the TDHF approach. As an example, 


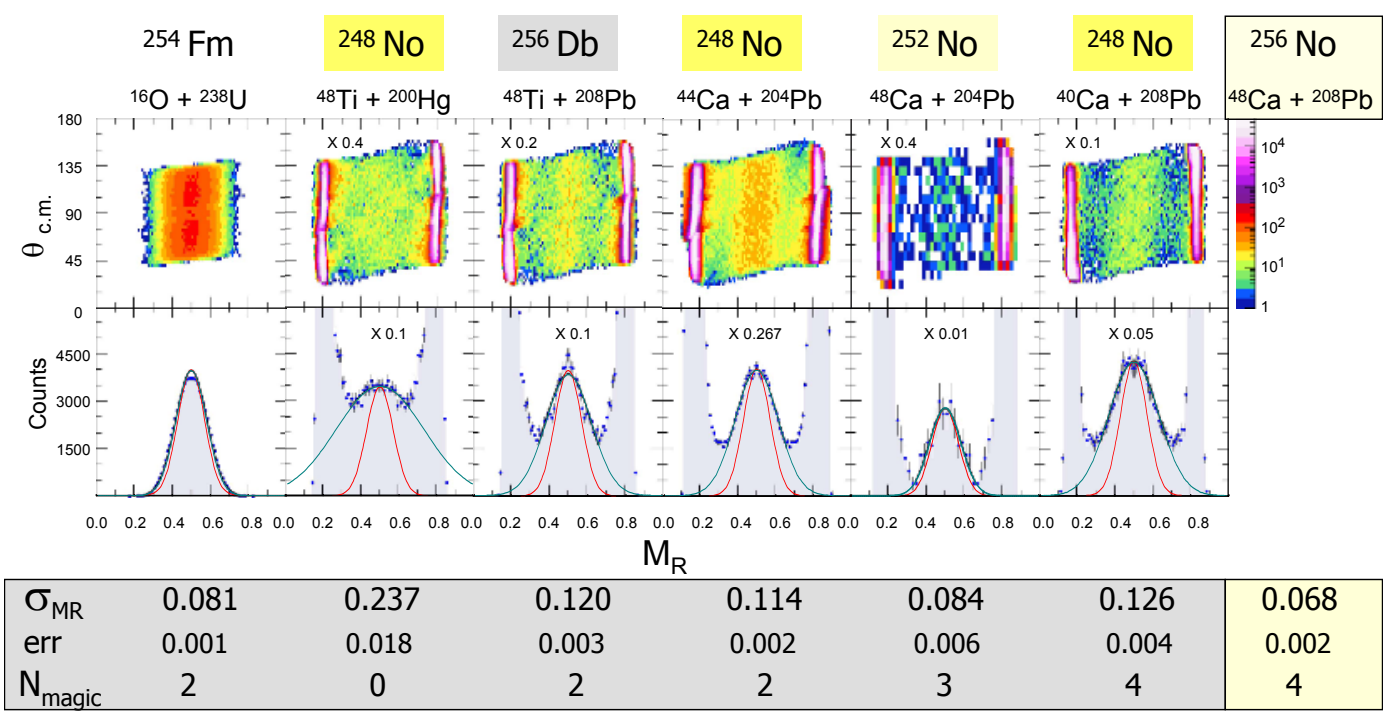

Figure 17. Measured mass-angle distributions for the indicated reactions (upper panels). The factor multiplies the maximum counts of the logarithmic color scale (right). In the projected mass ratio spectra (lower panels) the scale factor multiplies the counts scale on the left. Gaussian fits to the region around $M_{R}=0.5$ are shown (turquoise lines), whose standard deviations $\sigma_{M R}$ are given in the table below. Gaussian functions with $\sigma_{M R}=0.07$ (thin red lines) are shown for reference. The number of magic numbers in the entrance channels are indicated for each reaction.

TDHF calculations predict a range of times up to $2 \times 10^{-20} \mathrm{~s}$ in ${ }^{40,48} \mathrm{Ca}+{ }^{238} \mathrm{U}$ reactions [69, 70]. Experimental methods to obtain quantitative timescales [71] for fission reactions include pre-scission neutron multiplicities [72-74], crystal blocking [75, 76], and observation of compound nucleus X-rays [77]. Each has problems when faced with measuring the short quasifission timescales [77, 78]. The MAD offers the clearest sensitivity [59] to timescales of $\sim 10^{-20} \mathrm{~s}$. Mass angle distributions were measured for the reactions of ${ }^{34} \mathrm{~S}+{ }^{186} \mathrm{~W},{ }^{48} \mathrm{Ti}+{ }^{186} \mathrm{~W}$ and ${ }^{64} \mathrm{Ni}+{ }^{184} \mathrm{~W}$, for a range of beam energies [79], to compare with crystal blocking measurements for reactions of the same elements. The latter were interpreted as giving mean quasifission times of $\sim 10^{-18} \mathrm{~s}$ for all reactions [75]. The measured MADs are shown in Fig. 19. The lower panels show the $\mathrm{M}_{R}$ projections for $45^{\circ} \leq \theta_{\text {c.m. }} \leq 135^{\circ}$. They show marked differences between the three reactions. The ${ }^{64} \mathrm{Ni}$ reaction gives a minimum in yield at symmetry $\left(\mathrm{M}_{R}=0.5\right)$, ${ }^{48} \mathrm{Ti}$ a broad peak around symmetry, whilst ${ }^{34} \mathrm{~S}$ gives a narrow peak. Together with the correlation of mass and angle seen in the MADs, quasifission with short average reaction times appears dominant in the ${ }^{64} \mathrm{Ni}$ and ${ }^{48} \mathrm{Ti}$ reactions. To obtain more quantitative reaction timescales for the dominant quasifission, a classical Monte Carlo MAD simulation was developed [79].

Using the time distributions shown in the top right panels of Fig. 19, the simulated MADs (shown below them) reproduce the features of the experimental MADs. The corresponding $M_{R}$ spectra for $45^{\circ} \leq \theta_{\text {c.m. }} \leq 135^{\circ}$ (lowest right-hand panels of Fig. 19) also agree. The mean scission time for quasifission is quite well defined for these reactions, at $5 \times 10^{-21} \mathrm{~s}$ for ${ }^{64} \mathrm{Ni}$, and $10 \times 10^{-21} \mathrm{~s}$ for ${ }^{48} \mathrm{Ti}$. For the ${ }^{34} \mathrm{~S}$ reaction, the reaction times (rotation angles) are too large to conclude more than that only a small frac- tion of fission occurs before $10 \times 10^{-21} \mathrm{~s}$. These results, when compared with the longer crystal blocking times, suggest the need for a consistent assessment of all experimental techniques for determination of fission times, including development of a complete and consistent analysis framework for pre-fission neutrons [72-74, 80, 81]. A coordinated series of measurements for the same reactions, using all the different time-sensitive techniques, would give valuable insights.

\section{Summary and Conclusions}

The improved understanding of nuclear fusion has revealed an unexpectedly intricate relationship between fusion dynamics and the nuclear structure of the colliding nuclei. This was found first in fusion (capture) barrier distributions, which show in a direct way the critical role played by the quantum superposition of the states of the two colliding nuclei that are excited at and around the barrier radius. The states that couple strongly depend entirely on the nuclear structure of the two colliding nuclei, which can often be "seen" directly in the structure of the barrier distribution.

In reactions of weakly bound nuclei, it was a surprise that transfer reactions populating short-lived states of neighbouring nuclei play a stronger role in the reaction dynamics of fusion, incomplete fusion and breakup than direct excitation above their own breakup thresholds. This has implications for both experimental and theoretical understanding of reactions of nuclei near the drip lines, and for nuclear astrophysics.

The transition from a quantum superposition to an irreversible merged system had been taken to be a "hidden" process inside the fusion barrier, whose details should not 

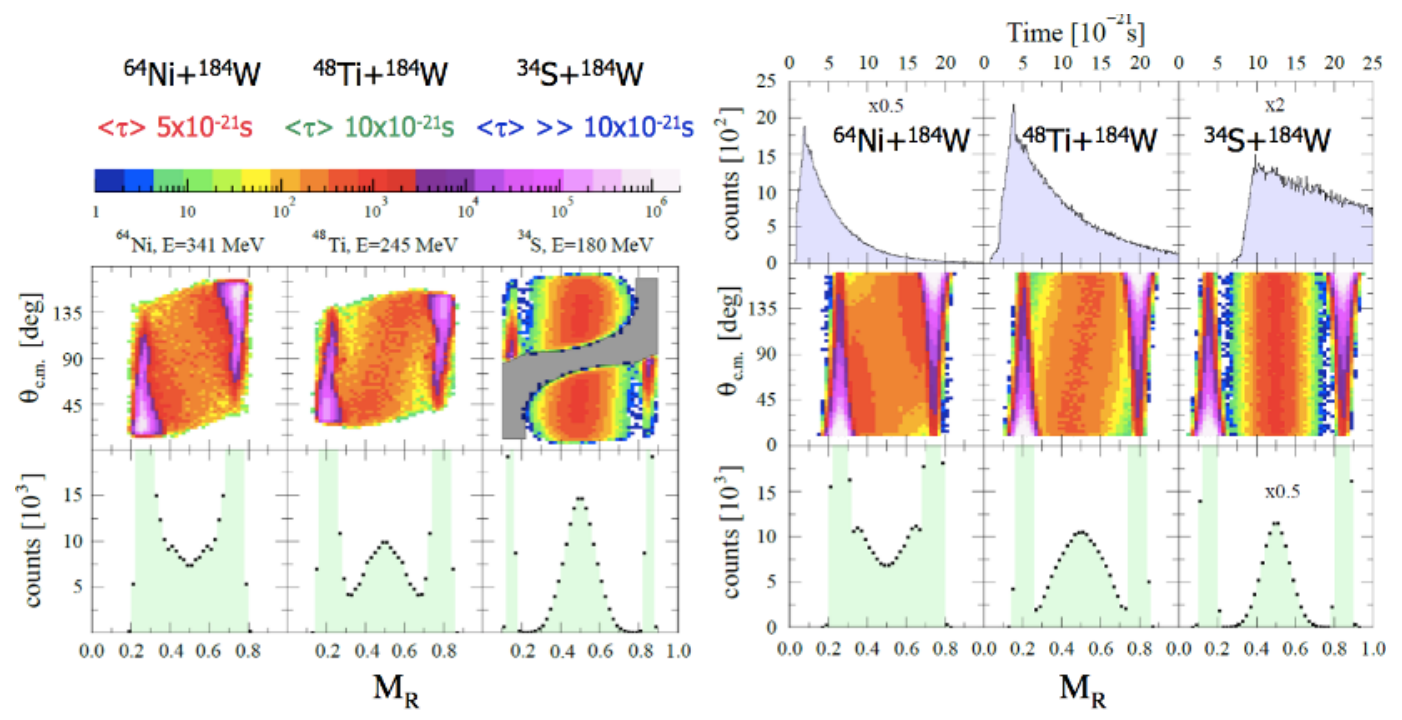

Figure 19. (Left panels) Experimental MADs for the reactions indicated, with the projected mass-ratio spectra below. They show very different behaviour for each reaction. (Right panels) Simulated MADs which reproduce the experimental MAD. The time distributions needed to match the experiments are shown above the simulations. The deduced mean reaction times for each measurement (shown at the top left) are quite different, as expected from the very different MADs.

affect observables. Increasing evidence is being found that this is not the case, and we may have to directly address dissipative tunnelling to fully understand fusion. Nuclear reactions are thus being linked to developments in foundation quantum mechanics.

Finally, in fusion reactions forming very heavy nuclei, at near-barrier beam energies the nuclear structure of the colliding nuclei can play a very strong role in determining the probabilities for true fusion leading to heavy element formation, and for the competing quasifission process. This seems to arise not only through the quantum superposition found around the barrier radius that results in deformation alignment at sub-barrier energies, but also through the effect of closed shells on the potential energy surface of the joined system and on the strength and nature of nuclear viscosity. This complicated interplay is a difficult problem to address, both experimentally and theoretically. Microscopic theoretical approaches, for example using TDHF and beyond-TDHF models, are already starting to give insights $[82,83]$ into fusion reactions forming very heavy nuclei.

\section{Acknowledgments}

The authors are grateful for the long-term contributions of D.C. Weisser, N. Lobanov, T. Kibèdi, and all the accelerator staff of the ANU Heavy Ion Accelerator who have provided the capabilities that have been crucial in pursuing this research program. The authors acknowledge support from the Australian Research Council, through Discovery Grants DP110102858, DP110102879, DP130101569 and Fellowships FL110100098 and FT120100760.

\section{References}

[1] I.I. Gontchar, D.J. Hinde, M. Dasgupta, J.O. Newton, Phys. Rev. C 69, 024610 (2004)

[2] I.I. Gontchar, D.J. Hinde, M. Dasgupta, C.R. Morton, J.O. Newton, Phys. Rev. C 73, 034610 (2006)

[3] L.R. Gasques, L.C. Chamon, D. Pereira, M.A.G. Alvarez, E.S. Rossi, C.P. Silva, B.V. Carlson, Phys. Rev. C 69, 034603 (2004)

[4] A.S. Umar, V.E. Oberacker, Phys. Rev. C 74, 021601 (2006)

[5] K. Washiyama, D. Lacroix, Phys. Rev. C 78, 024610 (2008)

[6] N. Bohr, J.A. Wheeler, Phys. Rev. 56, 426 (1939)

[7] K. Hagino, N. Takigawa, Prog. Theor. Phys. 128, 1061 (2012)

[8] P. Fröbrich, I.I. Gontchar, Phys. Rep. 292, 131 (1998)

[9] G. Pollarolo, A. Winther, Phys. Rev. C 62, 054611 (2000)

[10] C. Simenel, Phys. Rev. Lett. 106, 112502 (2011)

[11] I.J. Thompson, Comp. Phys. Rep. 7, 167 (1988)

[12] C.H. Dasso, S. Landowne, A. Winther, Nucl. Phys. A 405, 381 (1983)

[13] M. Dasgupta, D.J. Hinde, N. Rowley, A.M. Stefanini, Annu. Rev. Nucl. Part. Sci. 48, 401 (1998)

[14] N. Rowley, G.R. Satchler, P.H. Stelson, Phys. Lett. B 254, 25 (1991)

[15] J.X. Wei, J.R. Leigh, D.J. Hinde, J.O. Newton, R.C. Lemmon, S. Elfstrom, J.X. Chen, N. Rowley, Phys. Rev. Lett. 67, 3368 (1991)

[16] C.R. Morton, M. Dasgupta, D.J. Hinde, J.R. Leigh, R.C. Lemmon, J.P. Lestone, J.C. Mein, J.O. Newton, H. Timmers, N. Rowley et al., Phys. Rev. Lett. 72, 4074 (1994) 
[17] A.M. Stefanini, D. Ackermann, L. Corradi, D.R. Napoli, C. Petrache, P. Spolaore, P. Bednarczyk, H.Q. Zhang, S. Beghini, G. Montagnoli et al., Phys. Rev. Lett. 74, 864 (1995)

[18] J.R. Leigh, M. Dasgupta, D.J. Hinde, J.C. Mein, C.R. Morton, R.C. Lemmon, J.P. Lestone, J.O. Newton, H. Timmers, J.X. Wei et al., Phys. Rev. C 52, 3151 (1995)

[19] K. Hagino, N. Takigawa, M. Dasgupta, D.J. Hinde, J.R. Leigh, Phys. Rev. Lett. 79, 2014 (1997)

[20] K. Hagino, N. Takigawa, M. Dasgupta, D.J. Hinde, J.R. Leigh, Phys. Rev. C 55, 276 (1997)

[21] M.D. Rodríguez, M.L. Brown, M. Dasgupta, D.J. Hinde, D.C. Weisser, T. Kibèdi, M.A. Lane, P.J. Cherry, A.G. Muirhead, R.B. Turkentine et al., Nucl. Instrum. Methods Phys. Res. A 614, 119 (2010)

[22] J.F. Liang, et al., To be submitted (2014)

[23] R. Rafiei, D.J. Hinde, M. Dasgupta, D.C. Weisser, A.G. Muirhead, A.B. Harding, A.K. Cooper, H.J. Wallace, N.R. Lobanov, A. Wakhle et al., Nucl. Instrum. Methods Phys. Res. A 631, 12 (2011)

[24] A.J. Horsley, D.J. Hinde, M. Dasgupta, R. Rafiei, A. Wakhle, M. Evers, D.H. Luong, R. du Rietz, Nucl. Instrum. Methods Phys. Res. A 646, 174 (2011)

[25] I.P. Carter, M.L. Brown, M. Dasgupta, D.J. Hinde, M. Evers, D.H. Luong, A. Wakhle, E. Williams, EPJ Web of Conferences 35, 05003 (2012)

[26] F.D. Becchetti, J.A. Brown, K. Ashktorab, J.W. Janecke, W.Z. Liu, D.A. Roberts, R.J. Smith, J.J. Kolata, K. Larrikin, A. Morsad et al., Nucl. Instrum. Methods Phys. Res. B 56-57, Part 1, 554 (1991)

[27] F.D. Becchetti, M.Y. Lee, T.W. O’Donnell, D.A. Roberts, J.J. Kolata, L.O. Lamm, G. Rogachev, V. Guimaraes, P.A. DeYoung, S. Vincent, Nucl. Instrum. Methods Phys. Res. A 505, 377 (2003)

[28] D.J. Hinde, M. Dasgupta, J.R. Leigh, J.C. Mein, C.R. Morton, J.O. Newton, H. Timmers, Phys. Rev. C 53, 1290 (1996)

[29] C.R. Morton, A.C. Berriman, M. Dasgupta, D.J. Hinde, J.O. Newton, K. Hagino, I.J. Thompson, Phys. Rev. C 60, 044608 (1999)

[30] M. Dasgupta, D.J. Hinde, A. Diaz-Torres, B. Bouriquet, C.I. Low, G.J. Milburn, J.O. Newton, Phys. Rev. Lett. 99, 192701 (2007)

[31] K. Washiyama, K. Hagino, M. Dasgupta, Phys. Rev. C 73, 034607 (2006)

[32] L.R. Gasques, M. Evers, D.J. Hinde, M. Dasgupta, P.R.S. Gomes, R.M. Anjos, M.L. Brown, M.D. Rodríguez, R.G. Thomas, K. Hagino, Phys. Rev. C 76, 024612 (2007)

[33] M. Evers, M. Dasgupta, D.J. Hinde, L.R. Gasques, M.L. Brown, R. Rafiei, R.G. Thomas, Phys. Rev. C 78, 034614 (2008)

[34] M. Evers, D.J. Hinde, M. Dasgupta, D.H. Luong, R. Rafiei, R. du Rietz, Phys. Rev. C 81, 014602 (2010)

[35] D.J. Hinde, R.L. Ahlefeldt, R.G. Thomas, K. Hagino, M.L. Brown, M. Dasgupta, M. Evers, L.R. Gasques,
M.D. Rodriguez, Phys. Rev. C 76, 014617 (2007)

[36] J.O. Newton, R.D. Butt, M. Dasgupta, D.J. Hinde, I.I. Gontchar, C.R. Morton, K. Hagino, Phys. Lett. B 586, 219 (2004)

[37] J.O. Newton, R.D. Butt, M. Dasgupta, D.J. Hinde, I.I. Gontchar, C.R. Morton, K. Hagino, Phys. Rev. C 70, 024605 (2004)

[38] J.G. Keller, B.B. Back, B.G. Glagola, D. Henderson, S.B. Kaufman, S.J. Sanders, R.H. Siemssen, F. Videbaek, B.D. Wilkins, A. Worsham, Phys. Rev. C 36, 1364 (1987)

[39] M. Dasgupta, D.J. Hinde, J. Newton, K. Hagino, Prog. Theor. Phys. Suppl. 154, 209 (2004)

[40] D.J. Hinde, M. Dasgupta, Prog. Theor. Phys. Suppl. 154, 1 (2004)

[41] M. Evers, M. Dasgupta, D.J. Hinde, D.H. Luong, R. Rafiei, R. du Rietz, Phys. Rev. C 84, 054614 (2011)

[42] S. Szilner, L. Corradi, G. Pollarolo, S. Beghini, B.R. Behera, E. Fioretto, A. Gadea, F. Haas, A. Latina, G. Montagnoli et al., Phys. Rev. C 71, 044610 (2005)

[43] F.L.H. Wolfs, Phys. Rev. C 36, 1379 (1987)

[44] D.J. Hinde, R. du Rietz, M. Dasgupta, R.G. Thomas, L.R. Gasques, Phys. Rev. Lett. 101, 092701 (2008)

[45] G. Pollarolo, EPJ Web of Conferences 17, 08002 (2011)

[46] A. Diaz-Torres, D.J. Hinde, M. Dasgupta, G.J. Milburn, J.A. Tostevin, Phys. Rev. C 78, 064604 (2008)

[47] C. Simenel, Phys. Rev. Lett. 80, 044613 (2010)

[48] K. Sekigawa, K. Yabana, Phys. Rev. C 88, 014614 (2013)

[49] G. Scamps, D. Lacroix, Phys. Rev. C 87, 014605 (2013)

[50] M. Dasgupta, D.J. Hinde, R.D. Butt, R.M. Anjos, A.C. Berriman, N. Carlin, P.R.S. Gomes, C.R. Morton, J.O. Newton, A. Szanto de Toledo et al., Phys. Rev. Lett. 82, 1395 (1999)

[51] M. Dasgupta, D.J. Hinde, K. Hagino, S.B. Moraes, P.R.S. Gomes, R.M. Anjos, R.D. Butt, A.C. Berriman, N. Carlin, C.R. Morton et al., Phys. Rev. C 66, 041602 (2002)

[52] M. Dasgupta, P.R.S. Gomes, D.J. Hinde, S.B. Moraes, R.M. Anjos, A.C. Berriman, R.D. Butt, N. Carlin, J. Lubian, C.R. Morton et al., Phys. Rev. C 70, 024606 (2004)

[53] R. Rafiei, R. du Rietz, D.H. Luong, D.J. Hinde, M. Dasgupta, M. Evers, A. Diaz-Torres, Phys. Rev. C 81, 024601 (2010)

[54] D.H. Luong, M. Dasgupta, D.J. Hinde, R. du Rietz, R. Rafiei, C.J. Lin, M. Evers, A. Diaz-Torres, Phys. Lett. B 695, 105 (2011)

[55] A. Diaz-Torres, D.J. Hinde, J.A. Tostevin, M. Dasgupta, L.R. Gasques, Phys. Rev. Lett. 98, 152701 (2007)

[56] A. Diaz-Torres, Comp. Phys. Commun.. 182, 1100 (2011)

[57] B.B. Back, Phys. Rev. C 31, 2104 (1985) 
[58] B.B. Back, R.R. Betts, J.E. Gindler, B.D. Wilkins, S. Saini, M.B. Tsang, C.K. Gelbke, W.G. Lynch, M.A. McMahan, P.A. Baisden, Phys. Rev. C 32, 195 (1985)

[59] J. Tōke, B. Bock, G.X. Dai, A. Gobbi, S. Gralla, K.D. Hildenbrand, J. Kuzminski, W. Müller, A. Olmi, H. Stelzer, Nucl. Phys. A 440, 327 (1985)

[60] A.C. Berriman, D.J. Hinde, M. Dasgupta, C.R. Morton, R.D. Butt, J.O. Newton, Nature 413, 144 (2001)

[61] D.J. Hinde, M. Dasgupta, A. Mukherjee, Phys. Rev. Lett. 89, 282701 (2002)

[62] W.Q. Shen, J. Albinski, A. Gobbi, S. Gralla, K.D. Hildenbrand, N. Herrmann, J. Kuzminski, W.F.J. Müller, H. Stelzer, J. Tōke et al., Phys. Rev. C 36, 115 (1987)

[63] D.J. Hinde, M. Dasgupta, J.R. Leigh, J.P. Lestone, J.C. Mein, C.R. Morton, J.O. Newton, H. Timmers, Phys. Rev. Lett. 74, 1295 (1995)

[64] D.J. Hinde, R. du Rietz, M. Dasgupta, R.G. Thomas, L.R. Gasques, Phys. Rev. Lett. 101, 092701 (2008)

[65] K. Nishio, H. Ikezoe, S. Mitsuoka, I. Nishinaka, Y. Nagame, Y. Watanabe, T. Ohtsuki, K. Hirose, S. Hofmann, Phys. Rev. C 77, 064607 (2008)

[66] C. Simenel, D.J. Hinde, R. du Rietz, M. Dasgupta, M. Evers, C.J. Lin, D.H. Luong, A. Wakhle, Phys. Lett. B 710, 607 (2012)

[67] E.V. Prokhorova, A.A. Bogachev, M.G. Itkis, I.M. Itkis, G. Knyazheva, N.A. Kondratiev, E.M. Kozulin, L. Krupa, Y.T. Oganessian, I.V. Pokrovsky et al., Nucl. Phys. A 802, 45 (2008)

[68] D.J. Hinde, M. Dasgupta, Phys. Lett. B 622, 23 (2005)

[69] C. Simenel, EPJ A 48, 441 (2012)

[70] C. Simenel, Lecture Notes in Physics 875, 95 (2013)

[71] D. Jacquet, M. Morjean, Progress in Particle and Nuclear Physics 63, 155 (2009)
[72] D.J. Hinde, D. Hilscher, H. Rossner, B. Gebauer, M. Lehmann, M. Wilpert, Phys. Rev. C 45, 1229 (1992)

[73] D.J. Hinde, Nucl. Phys. A 553, 255c (1993)

[74] L. Donadille, E. Liatard, B. Benoit, F. Hanappe, L. Stuttge, G. Rudolf, E.M. Kozulin, Y.A. Lazarev, P. Desesquelles, L.A. Litnevsky et al., Nucl. Phys. A 656, 259 (1999)

[75] J.U. Andersen, J. Chevallier, J.S. Forster, S.A. Karamian, C.R. Vane, J.R. Beene, A. GalindoUribarri, J.G. del Campo, C.J. Gross, H.F. Krause et al., Phys. Rev. C 78, 064609 (2008)

[76] M. Morjean, D. Jacquet, J.L. Charvet, A. L'Hoir, M. Laget, M. Parlog, A. Chbihi, M. Chevallier, C. Cohen, D. Dauvergne et al., Phys. Rev. Lett. 101, 072701 (2008)

[77] M.O. Frégeau, D. Jacquet, M. Morjean, E. Bonnet, A. Chbihi, J.D. Frankland, M.F. Rivet, L. TassanGot, F. Dechery, A. Drouart et al., Phys. Rev. Lett. 108, 122701 (2012)

[78] D.J. Hinde, R. du Rietz, M. Dasgupta, EPJ Web of Conferences 17, 04001 (2011)

[79] R. du Rietz, D.J. Hinde, M. Dasgupta, R.G. Thomas, L.R. Gasques, M. Evers, N. Lobanov, A. Wakhle, Phys. Rev. Lett. 106, 052701 (2011)

[80] D.J. Hinde, R.J. Charity, G.S. Foote, J.R. Leigh, J.O. Newton, S. Ogaza, A. Chatterjee, Phys. Rev. Lett. 52, 986 (1984)

[81] D.J. Hinde, R.J. Charity, G.S. Foote, J.R. Leigh, J.O. Newton, S. Ogaza, A. Chatterjee, Phys. Rev. Lett. 53, 2275 (1984)

[82] D.J. Kedziora, C. Simenel, Phys. Rev. C 81, 044613 (2010)

[83] C. Simenel, C. Golabek, D.J. Kedziora, EPJ Web of Conferences 17, 09002 (2011) 